\title{
28 Resarch Soure of
}

\section{Study On The Effect of Tunicate Cellulose \\ Nanocrystals in The Preparation of Sodium Alginate-Based Enteric Capsule}

\section{Dezhong Xu}

Fujian Agriculture and Forestry University

\section{Yanan Cheng}

Fujian Agriculture and Forestry University

\section{Shuai Wu}

Fujian Agriculture and Forestry University

Qiuxia Zou

Fujian Agriculture and Forestry University

\section{Ajoy Kanti Mondal}

Fujian Agriculture and Forestry University

\section{Dengwen Ning}

Fujian Agriculture and Forestry University

Fang Huang ( $\nabla$ huangfanghg@gmail.com )

Fujian Agriculture and Forestry University https://orcid.org/0000-0002-3290-7788

\section{Research Article}

Keywords: Tunicate cellulose nanocrystal, Sodium alginate, Enteric-soluble, Capsule

Posted Date: November 10th, 2021

DOl: https://doi.org/10.21203/rs.3.rs-949624/v1

License: (c) (i) This work is licensed under a Creative Commons Attribution 4.0 International License. Read Full License

Version of Record: A version of this preprint was published at Cellulose on January 31st, 2022. See the published version at https://doi.org/10.1007/s10570-022-04445-5. 


\section{Abstract}

In this work, the tunicate cellulose nanocrystal (tCNC) was extracted from tunicate by bleaching and acid hydrolysis. It was used as filler in the preparation of sodium alginate-based enteric capsule. The addition of tCNC with high aspect ratio (65) rendered the enteric capsule excellent physical properties. Compared with the control sample, when the addition of tCNC was $10 \%(\mathrm{wt})$, the water contact angle of the capsule was enhanced by $46.0 \%$, the opacity was increased by $356.8 \%$, the maximum tensile stress was increased by $142.6 \%$, the modulus of elasticity was increased by $240.3 \%$, and the elongation at break was increased by $133.8 \%$. In the in vitro degradation experiments, the capsule hardly degraded in the gastric environment ( $\mathrm{pH}$ 1.2), while in the intestinal environment ( $\mathrm{pH}$ 6.8), the degradation became slower with the increase of tCNC content, which was consistent with the properties of enteric capsule. This research developed a new direction for the application of tCNC in the pharmaceutical material productions.

\section{Introduction}

In the food and pharmaceutical industries, the capsule encapsulation (Samakradhamrongthai et al. 2019) technology has been widely used. The capsules are less likely to be broken down by saliva in the mouth than tablets (Fathi et al. 2018; Grill et al. 2020; Yan et al. 2020), providing good protection from damage and protecting the digestive and respiratory organs. Generally, according to the dissolution location, the capsules can be divided into two categories, the gastric capsule and the enteric capsule. Unlike the gastric capsule, the enteric capsule does not disintegrate in the lower pH environment, i.e., the acidic stomach. Upon reaching the small intestine, the enteric capsule starts to disintegrate and releases the drug in the neutral pH environment. This could protect the stomach from drug-induced irritation (AlGousous and Langguth 2015).

Materials with good physical properties and biocompatibility are used to produce enteric capsules to maintain the stability of the medicine during the storage (Kathpalia et al. 2014). Currently in the market, the raw material for capsule manufacturing is mainly gelatin (derived from the skin and bone of animals such as pigs and cows). In addition, gelatin is prone to cross-linking (Digenis et al. 1994) and the high moisture content of the capsules leads to instability of the filled hygroscopic drugs (Duconseille et al. 2015). Recently, some non-gelatin-based capsules attracted interest in the pharmaceutical material industry. The most common alternatives are hydroxypropyl methylcellulose (HPMC) (Fu et al. 2020), pullulan (Ding et al. 2020) and starch-based capsules (Chen et al. 2020; Ji et al. 2017). They serve as good substitutes for gelatin, but have some limitations since they are not $\mathrm{pH}$-dependent and unstable in the enteral therapy. Sodium alginate (SA) is widely used in food, pharmaceutical, and bioengineering fields owing to its non-toxicity, stability, biocompatibility, and biodegradability (Abbasiliasi et al. 2019; Fayaz et al. 2009). Thus, SA has the potential to be used in the capsule production and could be an alternative of the traditional gelatin capsules. However, the mechanical properties of SA are weak, which makes it difficult to form capsule in the molding process. 
The tunicates cellulose nanocrystal (tCNC) is extracted from the mantle of tunicate (a kind of marine animal). The tCNCs are linear polymers with many $D$-glucopyranose rings linked by $\alpha$ - and $\beta-(1,4)$ glycosidic bonds. Recently, our research group has found that the crystallinity index (Crl) and aspect ratio of tCNC are $93.9 \%$ and 65.0 , respectively. However, the $\mathrm{Crl}$ and aspect ratio of the softwood nanocellulose are $70.9 \%$ and 10.5 , respectively. These high $\mathrm{Crl}$ and aspect ratio render the tCNC excellent physical strength (Cheng et al. 2020). Therefore, from this point of view, the tCNC might be used as enforcement agent in the SA capsule formation to increase the physical strength.

In this work, we applied the tCNC in the SA-based capsule using as a plasticizer. The prepared capsules were analyzed by mechanical characterizations, Fourier-transform infrared spectroscopy (FT-IR), and scanning electron microscope (SEM). The results showed that the tCNC was uniformly embedded into the SA matrix to form a rigid capsule. With the addition of tCNC, the hydrophobic property, opacity and tensile properties were significantly improved in the resulted capsule. In addition, the capsule was relatively stable in acidic medium but degraded well in neutral medium. Therefore, the SA-tCNC capsule is expected to load gastric irritant drug as enteric capsules. As far as we know, this is the first research on the effect of tCNC in the enteric capsule formation. This research would provide potential use of tCNC in the pharmaceutical material industry.

\section{Materials And Methods \\ 2.1 Materials}

Tunicate were purchased from Weihai Sea Food Market in Shandong, China. Treasure blue edible pigment was purchased from Wilton Industries, Inc. Dialysis bag was purchased from MYM biological technology Co., Ltd. Phenyl isocyanate was purchased from Xiya Reagent Co., Ltd. KBr, KOH, phosphate buffered solution (PBS), $\mathrm{NaClO}_{2}, \mathrm{H}_{2} \mathrm{SO}_{4}, \mathrm{CH}_{3} \mathrm{OH}$, acetic acid, glycerol, tetrahydrofuran, pyridine and sodium alginate (SA) were purchased from Shanghai Aladdin Biochemical Technology Co., Ltd. All the chemicals were used in this study without further purification.

\subsection{Methods}

\subsubsection{Preparation of tCNC}

The tunicate specimens were slit with a knife to remove the internal organs. The residual tunicate mantles were washed thoroughly with deionized water (DI water). After that, the mantles were soaked in a $5 \%(\mathrm{w} / \mathrm{v}) \mathrm{KOH}$ solution for $10 \mathrm{~h}$. Then, $300 \mathrm{~mL} 2 \%$ sodium chlorite solution and $5 \mathrm{~mL}$ of anhydrous acetic acid were mixed properly to make bleaching solution. The mantles were washed 3 times with DI water to neutral, and bleached with $300 \mathrm{~mL}$ bleaching solution at $70^{\circ} \mathrm{C}$ for 6 hours, and the bleach solution was changed every 2 hours. At last they were washed thoroughly and cut into small pieces, which were designated as tunicate cellulose. 
The tunicate cellulose was further hydrolyzed by sulfuric acid following a literature method (Tang et al. 2014). The tunicate cellulose was weighed $3 \mathrm{~g}$ in $105 \mathrm{~mL} 64 \%(\mathrm{v} / \mathrm{v})$ sulfuric acid solution and stirred continuously for $2 \mathrm{~h}$ at $45^{\circ} \mathrm{C}$. The reaction was terminated by adding excess ( 10 times) DI water, and the precipitate was collected by centrifugation at $8000 \mathrm{r} / \mathrm{min}$ for $30 \mathrm{~min}$. The precipitate was dialyzed in a dialysis bag with a cut-off molecular weight of 12000-14000 until the $\mathrm{pH}$ of the solution became neutral. After dialysis, the suspension was sonicated for $30 \mathrm{~min}$ and designated as tCNC.

\subsubsection{Gel permeation chromatography (GPC) analysis of tCNC and SA}

The gel permeation chromatography (GPC) (Waters, Polymer Standards Service, Milford, USA) was used to determine the weighted average molecular weight $(\mathrm{Mw})$ and degree of polymerization (DP) of tCNC and SA. Prior to the analysis, the ICNC and SA were derivatized by phenyl isocyanate based on a literature method (Hubbell and Ragauskas 2010). The tCNC/SA (15 mg) was weighed and placed in a test tube equipped with a micro-stirring bar. Then anhydrous pyridine $(4.00 \mathrm{~mL})$ and phenyl isocyanate $(0.50 \mathrm{~mL})$ were added and the tubes were capped with rubber film and reacted in an oil bath (DF101, Shanghai Qiuzuo Scientific Instrument Co., Ltd, China) at $70{ }^{\circ} \mathrm{C}$ for $72 \mathrm{~h}$. And then $1.00 \mathrm{~mL}$ of $\mathrm{CH}_{3} \mathrm{OH}$ was added to quench the reaction. The reaction solution was added dropwisely to a volume ratio of $7: 3 \mathrm{CH}_{3} \mathrm{OH} / \mathrm{H}_{2} \mathrm{O}$ solution $(100.0 \mathrm{~mL})$ to precipitate the reaction product. The solids were collected by filtration and then washed with methanol/water $(1 \times 50.0 \mathrm{~mL})$ followed by water $(2 \times 50.0 \mathrm{~mL})$. The derivatized $\mathrm{tCNC} / \mathrm{SA}$ was dried under vacuum at $40{ }^{\circ} \mathrm{C}$ for $12 \mathrm{~h}$. The derivatized tCNC and SA were then dissolved in tetrahydrofuran $(2 \mathrm{mg} / \mathrm{mL})$ for the GPC analysis. The DP is calculated by dividing the Mw of tCNC and SA by $519 \mathrm{~g} / \mathrm{mol}$ and $436 \mathrm{~g} / \mathrm{mol}$, which are the molecular weights of the derivatized cellulose repeating units and SA repeating units, respectively.

\subsubsection{Transmission electron microscopy (TEM) analysis of tCNC}

The morphology of tCNC was studied by TEM (TECNAI G2 F20, FEI Co., USA). One drop of tCNC suspension ( $1 \mathrm{wt} \%$ ) was deposited on the surface of a copper grid covered with a porous carbon film. The grids were then allowed to float in a $2 \mathrm{wt} \%$ uranyl acetate solution for 3 min to stain the samples. Finally, the samples were dried at room temperature for $24 \mathrm{~h}$ and then tested by TEM with a resolution of $0.2 \mathrm{~nm}$ at an accelerating voltage of $100 \mathrm{KV}$.

\subsubsection{Preparation of tCNC-SA film}

In order to perform the physical and mechanical properties testing of the tCNC-SA capsule, the tCNC-SA was firstly form a film since it has flat shape which is convenient for testing. The tCNC-SA film was formed as following: SA (3g) was firstly dissolved in DI water $(100 \mathrm{~mL})$ at $70^{\circ} \mathrm{C}$ in the water shaking bath (SY-2230, Crystal Instruments, USA). Then the glycerol $(0.75 \mathrm{~mL})$ was dropwisely added in the SA solution. After that, the tCNC was added to the solution in amounts of $0 \%, 1 \%, 3 \%, 5 \%$ and $10 \%$ of the total dry weight mass, which were marked as F0, F1, F2, F3 and F4, respectively. The tCNC was dispersed 
evenly in the SA solution by ultrasound and the tCNC-SA gel was formed. After that, the resulted gel was degased by water vacuum pump for 30 mins prior to the viscosity measurement. The viscosity of the tCNC-SA gel was determined by a viscometer (NDJ-5S, Shanghai Xingliang Optical Instrument Co., Ltd, China). In the meanwhile, the resulted gel was poured into a $90 \mathrm{~mm}$ diameter Petri dish and dried at $45^{\circ} \mathrm{C}$ for $24 \mathrm{~h}$ to form the tCNC-SA film. The preparation process is shown in Fig. 1.

\subsubsection{Mechanical properties measurement}

The mechanical properties (tensile strength, modulus of elasticity, elongation) of the tCNC-SA film were measured by universal material testing machine (Instron 3365, Instron Corporation, USA). The tCNC-SA film was cut into long strips of $62 \mathrm{~mm} \times 20 \mathrm{~mm}$ and the film thickness was measured by vernier calipers (Baigong 0-150mm, Shanghai Shenhan Measuring Tools Co., Ltd, China). The clamping distance was 25 $\mathrm{mm}$ and the stretching speed was $10 \mathrm{~mm} / \mathrm{min}$. Three parallel samples were measured and the average value was adopted. In addition, the tCNC-SA films (F0 and F3) were bended and twisted to visually observe its flexibility.

\subsubsection{Opacity measurement}

The opacity of the tCNC-SA film was determined by its UV absorbance based on a literature method (Abbasiliasi et al. 2019). Briefly, the tCNC-SA film was cut into squares with the size of $20 \mathrm{~mm} \times 20 \mathrm{~mm}$. The UV absorbances of these samples were recorded at $600 \mathrm{~nm}$ by UV spectrophotometer (Agilent 8453 , Agilent Technologies Co. Ltd, USA) to calculate the opacity with the following equation (1) (Siripatrawan and Harte 2010):

$$
\text { Opacity }=\frac{A}{t}
$$

Where $A$ is the UV absorbance of the film at $600 \mathrm{~nm}$, $\mathrm{t}$ is the film thickness, $\mathrm{mm}$.

\subsubsection{FT-IR analysis}

The SA, glycerol, tCNC, and tCNC-SA film samples were placed on a Fourier infrared spectrometer (VERTEX 70, Germany Brooke Technology Co., Ltd, Germany) to determine the infrared spectrum. The resolution was set to $4 \mathrm{~cm}^{-1}$ and the samples were scanned 32 times in the range of 4000 to $400 \mathrm{~cm}^{-1}$.

\subsubsection{X-ray diffraction analysis}

The X-ray diffraction (XRD) was used to analyze the crystallinity of the tCNC and tCNC-SA film samples. The scanning speed was set to $2^{\circ} / \mathrm{min}$ and scanned in the range of $2 \theta=5^{\circ} \sim 45^{\circ}$ with a voltage of $40 \mathrm{kV}$ and a current of $40 \mathrm{~mA}$.

According to the study of French (French 2014), the tCNC was judged to be cellulose $\mathrm{I}_{\beta}$. The crystallinity index (Crl) was calculated according to Segal's equation (2) (Segal et al. 1959; Yousefhashemi et al. 2019): 
$C r I=\frac{I_{200}-I_{a m}}{I_{200}} \times 100 \%$

Where $I_{200}$ is the diffraction intensity $\left(2 \theta=22.5^{\circ}\right)$ of the $(200)$ lattice plane in type I cellulose, and $I_{a m}$ is the diffraction intensity of the non-crystalline region at the minimum in the intensity near $18^{\circ}$.

\subsubsection{Preparation of tCNC-SA capsule}

The tCNC-SA capsules were prepared to observe the morphological changes in the in vitro degradation experiments. Basically, the tCNC-SA capsule and the tCNC-SA film shared the same chemical compositions. Their differences were on the formation process: the tCNC-SA capsule was dried in a mold while the tCNC-SA film was dried in a Petri dish.

The capsules were prepared following a reference method (Abbasiliasi et al. 2019). Preheat the stainless steel capsule mold at $70^{\circ} \mathrm{C}$ oven. Repeat the experimental step 2.2.4 to prepare tCNC-SA gel with different tCNC ratios, and $1 \%(\mathrm{wt})$ of treasure blue edible pigment was also added in the mixture. The formed gel was stirred well and was poured into the preheated mold. Finally, the mold was dried in $45^{\circ} \mathrm{C}$ oven for $4 \mathrm{~h}$. The amounts of 100 capsules were prepared by adding different proportions of tCNC film (F0-F4). The following equation (3) is used to calculate the capsule formation rate (C). Finally, the dried capsules were removed from the mold for subsequent characterization.

$C=\frac{N 1}{N 2} \times 100 \%$

Where $N 1$ is the number of capsules formed, and $N 2$ is the number of capsules made.

\subsubsection{Characterization of the tCNC-SA capsule}

The capsule characterization includes the appearance, the dry loss and water contact angle testing. The appearance of the tCNC-SA capsule was characterized by visual observation, and the dry loss was tested according to US Pharmacopoeia (USP) (Pharmacopeial 2016) method. The cap and body of the capsule were separated and weighed. After that, they were dried at $105^{\circ} \mathrm{C}$ oven for 6 hours and weighed the dry weight. The following equation (4) was used to calculate the dry loss.

$L=\frac{m 1}{m 2} \times 100 \%$

Where $m 1$ is the mass of the sample before drying, and $m 2$ is the mass of the sample after drying.

The capsule appearance includes surface smoothness, color uniformity, shape deformation and odor. The water contact angle testing of the capsules was described as following: at ambient temperature, a certain size of capsule $(20 \mathrm{~mm} \times 20 \mathrm{~mm})$ was cut and fixed on a carrier table, and the water of $5 \mu \mathrm{L}$ was dropped onto the capsule surface. The WCA was measured by goniometer (DSA 30, Kruss, Germany) to characterize the film hydrophobicity. Five points were tested for each sample and the average value was adopted. 


\subsubsection{Scanning electron microscopy (SEM) analysis}

The morphology of the tCNC-SA capsule was measured by SEM (Nova Nano SEM 230, FEI Co., USA). The treated sample was gold sprayed (100 s) by vacuum ion sputter (JFC-1600, JEOL Ltd, Japan), and the morphology of the sample was observed.

\subsubsection{In vitro degradation experiments of tCNC-SA capsule}

In order to protect the gastric mucosa from the irritation caused by the drug components, the capsules should not disintegrate and release drugs under acidic conditions. Therefore, in this research, the degradation study of the tCNC-SA was divided into two steps. The influences of tCNC loading and $\mathrm{pH}$ of PBS buffer solution on the capsule degradation were studied in the first and second step, respectively.

The first step focused on the effect of tCNC loading on capsule degradation time in a simulated human intestinal fluid of $\mathrm{pH} 6.8$ and temperature $\left(37^{\circ} \mathrm{C}\right)$. The optimized tCNC loading was based on the degradation time (60 min). In practice, each tCNC-SA capsule (F0, F1, F2, F3, F4) was weighed into glass test tubes, and $7 \mathrm{~mL}$ of $\mathrm{pH}$ 6.8 PBS was added separately and dissolved in a $37^{\circ} \mathrm{C}$ water shaking bath. The undissolved solid samples were collected through filteration at different time intervals $(0 \mathrm{~min}, 15 \mathrm{~min}$, $30 \mathrm{~min}$ and $60 \mathrm{~min}$ ). The sample weight loss during the dissolution was calculated by the ratio of the initial sample weight (o.d. weight) and the o.d. weight of the sample in a certain time interval.

In the second step, the optimized tCNC loading was applied in the tCNC-SA capsule formation. These capsules were used to study the pH effect on their degradation. Two pHs, 1.2 and 6.8 were chosen to simulate the human gastric and intestinal fluid environment, respectively (Ilgin et al. 2020). In this step, the capsules were dissolved in two PBS $(\mathrm{pH} 1.2$ and $\mathrm{pH} 6.8)$ at $37^{\circ} \mathrm{C}$. During this process, the undissolved capsules were collected and the weight losses were also calculated, as described in the first step.

\section{Results And Discussion}

\subsection{GPC analysis of SA and tCNC}

The Mw,Mn, DP and DPI of tCNC and SA are summarized in Table 1. The Mw of tCNC is $2.49 \times 10^{6} \mathrm{~g} / \mathrm{mol}$, $\mathrm{Mn}$ of of tCNC is $1.28 \times 10^{6} \mathrm{~g} / \mathrm{mol}$, the DP is 4672 and DPI is 1.95 . While the Mw of SA is $2.97 \times 10^{5}$ $\mathrm{g} / \mathrm{mol}, \mathrm{Mn}$ of $\mathrm{SA}$ is $1.69 \times 10^{5} \mathrm{~g} / \mathrm{mol}, \mathrm{DP}$ is 681 and DPI is 1.76 .

Table 1

$\mathrm{Mw}, \mathrm{Mn}, \mathrm{DP}$ and DPI of tCNC and SA.

\begin{tabular}{|lllll|}
\hline Sample & $\mathrm{Mw}(\mathrm{g} / \mathrm{mol})$ & $\mathrm{Mn}(\mathrm{g} / \mathrm{mol})$ & $\mathrm{DP}$ & $\mathrm{DPI}$ \\
\hline $\mathrm{tCNC}$ & $2.49 \times 10^{6}$ & $1.28 \times 10^{6}$ & 4798 & 1.95 \\
\hline $\mathrm{SA}$ & $2.97 \times 10^{5}$ & $1.69 \times 10^{5}$ & 681 & 1.76 \\
\hline
\end{tabular}




\subsection{TEM analysis of tCNC}

As shown in the TEM image (Fig. 2), the length and width of the tCNC were measured as $1300 \mathrm{~nm}$ and 20 $\mathrm{nm}$, respectively. Then the aspect ratio was calculated as 65 , which is in the range of previous report (Peng et al. 2011).

\subsection{Formation of tCNC-SA film/capsule}

During the tCNC-SA film/capsule formation, the hydrogen bonding is formed between the hydroxyl groups in SA and the hydroxyl groups in tCNC, which forms a network structure (Fig. 3a). The glycerol is inserted between the molecular chains of SA and tCNC as a plasticizer. The intermolecular forces between the cellulose and SA molecular chains can be weakened by the glycerol, and the distance between them is increased (Xu et al. 2012). As a result, the possibility of movement between SA and the tCNC is increased, and the entanglement between molecular chains is reduced, resulting in increased plasticity of the composites.

The viscosity of the tCNC-SA gel is demonstrated in Fig. 3b. As the tCNC content increases, the viscosity of the tCNC-SA film gradually increases from $1.28 \mathrm{~Pa}$ s to $1.69 \mathrm{~Pa} \mathrm{~s}$, which is $31.32 \%$ higher than that of blank sample F0. This phenomenon is attributed to the hydrogen bonding between tCNC and SA (Salas et al. 2014).

\subsection{Mechanical property analysis of tCNC-SA film}

Five stress-strain curves of tCNC-SA films are shown in Fig. 4a. There is basically no residual strain after the fracture, and the section is perpendicular to the direction of stress, indicating the film has a certain degree of rigidity and toughness (Revin et al. 2019). As the content of tCNC increases from 0 wt\% to 10 $w t \%$, the tensile stress of the tCNC-SA film increases from $12.64 \mathrm{MPa}$ to $30.67 \mathrm{MPa}$, with a maximum increase of $142.6 \%$. Fig. $4 \mathrm{~b}$ shows the elastic modulus of different tCNC-SA films. With the addition of tCNC, compared with the control F0 sample, the elastic modulus of the film increases up to $240.3 \%$ when it contains $10 \%$ of tCNC (sample F4). Generally, the higher the modulus of elasticity, the more rigid the sample is and the less likely to be deformed. The improvements of the tensile stress and elastic modulus of the tCNC-SA film are due to the inherent advantages of high aspect ratio and high elastic modulus of the tCNCs, which shows excellent reinforcement in the polymer matrix (Iwamoto et al. 2009). When the tCNC was applied in the capsule formation, it has a certain degree of resistance to compression, which is conducive to alleviating the extrusion of the capsule during production, handling and storage.

When tCNC were added at $1 \%$ (sample F1), the elongation of the film before fracture is increased by $100 \%$ compared to F0, as shown in the Fig. 4c. In a previous study by our group, it was found that tCNC had significant high aspect ratio of 65 , which renders it forming mesh-like structure in the composite. The high aspect ratio of the tCNC could enhance the binding sites between tCNC and SA (Boufi et al. 2016; Ming et al. 2021), forming interweaving network in the film. That might be the reason that the increase the tCNC content in the film could improve the elongation, as shown in Fig. 4c. It should be noted that, 
when the adding amounts increased from the $1 \%$ (sample F1) to $10 \%$ (sample F4), the film elongation rate was increased from $100-144 \%$ when compared with the contrast F0 sample. The results indicate the small quantity of tCNC could significantly increase the mechanical strength of the tCNC-SA. Further addition of tCNC has limit strength enhancement for the film. Furthermore, the addition of tCNC could also improve the film flexibility, as shown in Fig. 4 (d, e, f, g, h and i). The film made from the pure SA (sample F0) was rigid. Upon the bending and twisting force, it recovered to the original form rapidly. In contrast, the tCNC enhanced film (sample F3) is soft and flexible. The flexibility and softness is important for the capsules since these properties could reduce the irritation to the mucosa during the deglutition (Kathpalia et al. 2014)

The elasticity modulus and elongation rate of the enteric capsule are compared with previous report, as shown in Table 2. In current work, the elasticity modulus is much higher than that of HPMC/Gelatin and $\mathrm{NCC} /$ Gelatin, and the rate of elongation at break is slightly higher than that of HPMC/Gelatin. This indicates the mechanical properties of the capsule prepared in this study are superior or comparable to the reported capsules (Bae et al. 2008; Jimenez et al. 2010; Zhang et al. 2017).

Table 2

The comparisons of the elasticity modulus and elongation rate of different enteric capsules

\begin{tabular}{|llll|}
\hline $\begin{array}{l}\text { Filler/main } \\
\text { ingredient }\end{array}$ & $\begin{array}{l}\text { Elasticity modulus } \\
\text { (MPa) }\end{array}$ & $\begin{array}{l}\text { Rate of elongation at } \\
\text { break (\%) }\end{array}$ & Reference \\
\hline tCNC/SA & $607.7-1789.7$ & $4.6-10.9$ & This work \\
\hline HPMC/Gelatin & $168-255$ & 9.3 & $\begin{array}{l}\text { (Bae et al. 2008; Jimenez et } \\
\text { al. 2010) }\end{array}$ \\
\hline NCC/Gelatin & $56-610$ & - & (Zhang et al. 2017) \\
\hline
\end{tabular}

\subsection{Physical properties of tCNC-SA capsules}

Physical properties of the capsule mainly include appearance, opacity, testing of water contact angle and the SEM observations. Table 3 summarizes the appearance, dry loss and formation rate data of capsules with different addition of tCNC. Without the addition of tCNC, the capsule formation rate was 0 (sample F0) since there is no complete tCNC-SA capsule formed in the mold. Upon the visually observation, the formed capsules (F1 to F4) have smooth surface with no cracks, no air bubbles and no odor.

As the amount of tCNC increases, the capsule formation rate gradually increases. The capsule formation rate reaches $92 \%$ when the amount of $\mathrm{tCNC}$ increases to $10 \%$. The addition of $\mathrm{tCNC}$ renders the enhancement of physical strength and flexibility of the SA film, eventually leading to the increase of the capsule formation rate.

The dry loss of F0 was only $8.61 \%$ and reached a maximum of $15.45 \%$ (sample F4) with the $10 \mathrm{wt} \%$ of tCNC added. This is due to the hydrogen bonding between tCNC and SA, which results in the absorption 
of water (Gilormini and Verdu 2018) in the prepared film and therefore the dry loss increases.

It can be visually observed in Fig. 5a and Fig. 5c that the opacity of the tCNC-SA capsule increases with the increase of the tCNC content. The F0 is a SA film with an opacity of 0.44 and high transparency. When $1 \%$ tCNC and $10 \%$ tCNC were added (Sample F1 and F4), the increases of opacity are $15.9 \%$ and $356.8 \%$, respectively. This is due to the reason that the tCNC is partially agglomerated in the film and forms a self-assembled network, which hinders the passage of light through the film (Qian et al. 2010). Since all the film thickness are similar as discussed in the sample preparation, the film opacity is directly related to the film UV absorbance, as indicated in Equation 1. According to the opacity data in Table 3, the tCNC-SA film opacities increases with the increase of tCNC content, which means that the film material has the potential to act as a UV absorber, rendering it light sheltering abilities. Therefore, the adding of tCNC in the capsule could reduce the amount of shading agent added during the preparation of capsules.

Table 3

Physical properties of tCNC-SA capsules

\begin{tabular}{|lllllc|}
\hline Testing items & \multicolumn{5}{l}{ Samples } \\
\cline { 2 - 6 } & F0 & F1 & F2 & F3 & F4 \\
\hline Appearance & - & \multicolumn{2}{l|}{ No cracks, smooth surface, no bubbles and no odor } \\
\hline Dry Loss (\%) & 8.61 & 12.01 & 13.16 & 15.15 & 15.45 \\
\hline Capsule formation rate (\%) & 0 & 76 & 84 & 90 & 92 \\
\hline
\end{tabular}

The WCA is used to determine the resistance of the tCNC-SA capsule surface to liquid water, which can reflect the hydrophobicity/hydrophilicity of the sample surface (Rhim 2011). The pure SA film (sample F0) has poor water resistance, and the addition of tCNC can improve this hydrophobicity, as shown in Fig. $5 \mathrm{~b}$. There is no $\mathrm{tCNC}$ in the F0 sample, which has a WCA of $36.5^{\circ}$, and the water diffuses randomly in all directions. As the tCNC content increases, the WCA of the film becomes enlarged, which also leads to a more circular water permeation shape. This indicates that the addition of tCNC makes the propagation of water resistance more uniform. When the addition of tCNC was $10 \%$, the water contact angle of the film surface was $53.3^{\circ}$, which increased by $46.0 \%$ compared with $0 \%$ tCNC addition sample. A strong hydrogen bonding can occur between the hydroxyl group on tunicate nanocellulose and the hydroxyl groups in SA, thus improving the bonding of these two substrates and inhibiting the water absorption of the sample. After wetting, water molecules cannot break through these strong hydrogen bonds, so it plays a role in improving the hydrophobic property of the film (Abdollahi et al. 2013).

The ratios of tCNC are different, and the surface structure of the tCNC-SA capsule is also different. The surface and cross-section of the tCNC-SA films were characterized using SEM to investigate the morphology of tCNC in the SA substrate film, as shown in Fig. 6. From the surface diagram (a-e), it can be observed that tCNC with high aspect ratio exists in the film in the form of thin strips. From F0 to F4, the 
tCNC is added more and more, the surface of the film becomes rougher and even some agglomeration occurs. In the cross-sectional view (Fig. 6: h-j), it is observed that tCNC is uniformly distributed in the film in the form of network, which could enhance the physical properties of the film. This is the reason why the mechanical properties of tCNC-CA films become stronger as the amount of tCNC increases.

\subsection{Chemical characterization of tCNC-SA film}

Figure 7a represents the FT-IR spectra of tCNC,glycerol, SA, F0, F1, F2, F3 and F4. No chemical reaction occurred during the preparation of the tCNC-SA film, so no new characteristic peaks appear in F0, F1, F2, $\mathrm{F} 3$ and F4. And the transmittance of F0, F1, F2, F3 and F4 in the IR spectrum increases with the increase of tCNC content. The absorption peaks at $3415 \mathrm{~cm}^{-1}, 3342 \mathrm{~cm}^{-1}, 1638 \mathrm{~cm}^{-1}$ and $1413 \mathrm{~cm}^{-1}$ are attributed to the $0-\mathrm{H}$ stretching vibration. The absorption peaks at $2920 \mathrm{~cm}^{-1}$ and $2904 \mathrm{~cm}^{-1}$ is attributed to the $\mathrm{C}-\mathrm{H}$ stretching vibration. The absorption peak at $1620 \mathrm{~cm}^{-1}$ is attributed to $\mathrm{C}=\mathrm{C}$ stretching vibration. The two absorption peaks at $1299 \mathrm{~cm}^{-1}$ and $1089 \mathrm{~cm}^{-1}$ are caused by $\mathrm{C}-\mathrm{O}$ stretching vibration, and the absorption peak at $817 \mathrm{~cm}^{-1}$ is attributed to the $\mathrm{C}=\mathrm{C}$ bending vibration (Saravanakumar et al. 2020). Two characteristic peaks of glycerol at $2937 \mathrm{~cm}^{-1}$ and $2879 \mathrm{~cm}^{-1}$ belong to $\mathrm{CH}_{2}$ asymmetric and symmetric stretching vibration, respectively. The characteristic peaks of tCNC, SA, and glycerol interact with each other, which results in peak overlaps in two large ranges $\left(3778 \mathrm{~cm}^{-1}-2437 \mathrm{~cm}^{-1}\right.$ and 1856 $\mathrm{cm}^{-1}-1016 \mathrm{~cm}^{-1}$ ) (Zhao et al. 2020).

Figure 7b shows the XRD spectrum of the crystal structure of the tCNC and tCNC-SA films (samples F0 to F4). It can be seen that the diffraction characteristics of the films (F1, F2, F3 and F4) are essentially the same as those of $\mathrm{tCNC}$, which is a typical cellulose l-type structure with three typical lattice surfaces with intensities of $2 \theta=14.66^{\circ}(1-10), 16.58^{\circ}(110)$ and $22.76^{\circ}(200)$, respectively (Cheng et al. 2020; Narita et al. 2005). The calculated $\mathrm{Cr}$ /values of F1, F2, F3, F4 and tCNC are $32.4 \%, 57.3 \%, 68.8 \%, 82.1 \%$ and $96.1 \%$, respectively. The F0 results show that SA had no obvious crystalline features, indicating the amorphous structure of alginate. In the film samples (FO to F4), the intensity of the characteristic peaks gradually increases with the increase of tCNC.

\subsection{Degradation of tCNC-SA capsule}

\subsubsection{Degradation of tCNC-SA capsule with different tCNC ratios}

The addition of tCNC inhibited the degradation of the capsule as analyzed in Fig. 8a and Fig. 8b. F1, F2, F3 and F4 disintegrate at 15 mins without affecting the release of drug, which is in accordance with the US Pharmacopoeia requirements on disintegration time of hard capsules (Pharmacopeial 2016). F1 degrades completely within 60 min, F2, F3 and F4 cannot be completely degraded within 60 min, especially F4, where the mass loss value is about $50 \%$ at $60 \mathrm{~min}$. This is due to the fact that tCNC and SA are cross-linked by hydrogen bonds and form a mesh structure, which hinder the disintegration of the capsules. Therefore, with the increase of tCNC content, the mass loss is smaller. 


\subsubsection{In vitro degradation of tCNC-SA capsule in different pH environments}

Figure 9a shows the in vitro degradation mass loss and degradation photos of $\mathrm{F} 2$ at different $\mathrm{pH}$ environments ( $\mathrm{pH} 1.2$ and pH 6.8). The mass loss of the tCNC-SA capsule in $\mathrm{pH} 6.8$ increases continuously with time and finally reached $67.3 \%$ at $60 \mathrm{~min}$. In contrast, in the solution with $\mathrm{pH}=1.2$, the mass loss of the tCNC-SA capsule is $8.2 \%$ after $30 \mathrm{~min}$, and then the mass is stabilized (Fig. 9b). The SA contains a large amount of $-\mathrm{COO}^{-}$. Under acidic conditions, it is turned into $-\mathrm{COOH}$. Thus, the ionization and the hydrophilicity of SA decrease, leading to the contraction of molecular chains (Hua et al. 2010). Therefore, the prepared tCNC-SA capsule hardly degrades in acidic conditions. The capsule in pH 6.8 is softened at $15 \mathrm{~min}$ and cannot maintain their original shape, and most of them degrade at $60 \mathrm{~min}$. Based on these observations, the tCNC-SA capsule can be applied as enteric-soluble capsule, which is loaded with drugs that are irritating to the stomach. As a result, the tCNC-SA capsule can be digested and absorbed in the intestine.

\section{Conclusions}

In this study, the tCNC enhanced SA-based capsules were prepared. Due to the formation of hydrogen bonds between tCNC and SA, the mechanical properties, water contact angle, opacity of the tCNC-SA capsules are significantly improved. When tCNC loading was $10 \%(\mathrm{w} / \mathrm{w})$, the water contact angle of the capsules increased by $46.0 \%$, opacity increased by $356.8 \%$, tensile stress increased by $142.6 \%$, elastic modulus increased by $240.3 \%$, and elongation increased by $137.0 \%$, respectively.

During the in vitro disintegration experiment, the conversion of $-\mathrm{COO}^{-}$in $\mathrm{SA}$ to $-\mathrm{COOH}$ under acidic conditions leads to decrease of capsule ionization. As a result, the tCNC-SA capsules barely disintegrated under acidic conditions ( $\mathrm{pH}$ 1.2), while they could disintegrate at a pH of 6.8 in $15 \mathrm{~min}$, which indicates the excellent $\mathrm{pH}$ responsibilities.

Therefore, the prepared tCNC-SA composite is expected to be used as enteric capsules, which lays the foundation for the application of tCNC in pharmaceutical material production.

\section{Declarations}

\section{CRediT authorship contribution statement}

Dezhong Xu: Conceptualization, Methodology, Validation, Formal analysis, Investigation. Yanan Cheng: Conceptualization, Methodology, Validation, Formal analysis, Investigation. Shuai Wu: Resources, Data curation, Writing - original draft. Qiuxia Zou \& Ajoy Kanti Mondal: Methodology, Validation, Formal analysis, Investigation. Dengwen Ning: Writing - review \& editing, Visualization. Fang Huang: Supervision, Writing - review \& editing, Project administration, Funding acquisition. 


\section{Declaration of Competing Interest}

The authors report no declarations of interest.

\section{Acknowledgements}

This work was supported by National Key Research and Development Program of China (2017YFB0307900), National Natural Science Foundation of China (22078061), the guide project from Department of Science and Technology of Fujian Province (2018H0006), and the Foundation (KF201922) of State Key Laboratory of Biobased Material and Green Papermaking, Qilu University of Technology, Shandong Academy of Sciences.

\section{References}

1. Abbasiliasi S, Tan JS, Ibrahim T, Ismail N, Ariff AB, Mokhtar NK, Mustafa S (2019) Use of sodium alginate in the preparation of gelatin-based hard capsule shells and their evaluation in vitro. RSC Adv 9:16147-16157. https://doi.org/10.1039/C9RA01791G

2. Abdollahi M, Alboofetileh M, Behrooz R, Rezaei M, Miraki R (2013) Reducing water sensitivity of alginate bio-nanocomposite film using cellulose nanoparticles. Int J Biol Macromo 54:166-173. https://doi.org/10.1016/j.jibiomac.2012.12.016

3. Al-Gousous J, Langguth P (2015) European versus United States Pharmacopeia Disintegration Testing Methods for Enteric-Coated Soft Gelatin Capsules. Dissolut Technol 22:6-8. https://doi.org/10.14227/DT220315P6

4. Bae HJ, Cha DS, Whiteside WS, Park HJ (2008) Film and pharmaceutical hard capsule formation properties of mungbean, waterchestnut, and sweet potato starches. Food Chem 106:96-105. https://doi.org/10.1016/j.foodchem.2007.05.070

5. Boufi S, Gonzalez I, Delgado-Aguilar M, Tarres Q, Angels Pelach M, Mutje P (2016) Nanofibrillated cellulose as an additive in papermaking process: A review. Carbohyd Polym 154:151-166. https://doi.org/10.1016/j.carbpol.2016.07.117

6. Chen Q, Zong Z, Gao X, Zhao Y, Wang J (2020) Preparation and characterization of nanostarchbased green hard capsules reinforced by cellulose nanocrystals. Int J Biol Macromo 167:1241-1247. https://doi.org/10.1016/j.jibiomac.2020.11.078

7. Cheng Y, Mondal AK, Wu S, Xu D, Ning D, Ni Y, Huang F (2020) Study on the Anti-Biodegradation Property of Tunicate Cellulose. Polymers 12:3071. https://doi.org/10.3390/polym12123071

8. Digenis GA, Gold TB, Shah VP (1994) Cross-linking of gelatin capsules and its relevance to their in vitro-in vivo performance. J Pharm Sci 83:915-921. https://doi.org/10.1002/jps.2600830702

9. Ding Y, Jiang F, Chen L, Lyu W, Chi Z, Liu C, Chi Z (2020) An Alternative Hard Capsule Prepared with the High Molecular Weight Pullulan and Gellan: Processing, Characterization, and In Vitro Drug Release. Carbohyd Polym 237:116172. https://doi.org/10.1016/j.carbpol.2020.116172 
10. Duconseille A, Astruc T, Quintana N, Meersman F, Sante-Lhoutellier V (2015) Gelatin structure and composition linked to hard capsule dissolution: A review. Food Hydrocolloid 43:360-376. https://doi.org/10.1016/j.foodhyd.2014.06.006

11. Fathi M, Kazemi S, Zahedi F, Shiran MR, Moghadamnia AA (2018) Comparison of oral bioavailability of acetaminophen tablets, capsules and effervescent dosage forms in healthy volunteers. Curr Iss Pharm Med S 31:5-9. https://doi.org/10.1515/cipms-2018-0001

12. Fayaz AM, Balaji K, Girilal M, Kalaichelvan PT, Venkatesan R (2009) Mycobased Synthesis of Silver Nanoparticles and Their Incorporation into Sodium Alginate Films for Vegetable and Fruit Preservation. J Agric Food Chem 57:6246-6252. https://doi.org/10.1021/jf900337h

13. French AD (2014) Idealized powder diffraction patterns for cellulose polymorphs. Cellulose 21:885896. https://doi.org/10.1007/s10570-013-0030-4

14. Fu M, Blechar JA, Sauer A, Al-Gousous J, Langguth $P$ (2020) In Vitro Evaluation of Enteric-Coated HPMC Capsules-Effect of Formulation Factors on Product Performance. Pharmaceutics 12:696. https://doi.org/10.3390/pharmaceutics12080696

15. Gilormini P, Verdu J (2018) On the role of hydrogen bonding on water absorption in polymers. Polymer 142:164-169. https://doi.org/10.1016/j.polymer.2018.03.033

16. Grill S, Bruderer S, Sidharta PN, Antonova M, Globig S, Carlson J, Csonka D (2020) Bioequivalence of macitentan and tadalafil given as fixed-dose combination or single-component tablets in healthy subjects. Brit J Clin Pharmaco 86:2424-2434. https://doi.org/10.1111/bcp.14347

17. Hua S, Ma H, Li X, Yang H, Wang A (2010) pH-sensitive sodium alginate/poly (vinyl alcohol) hydrogel beads prepared by combined $\mathrm{Ca}^{2+}$ crosslinking and freeze-thawing cycles for controlled release of diclofenac sodium. Int J Biol Macromo 46:517-523. https://doi.org/10.1016/j.ijbiomac.2010.03.004

18. Hubbell CA, Ragauskas AJ (2010) Effect of acid-chlorite delignification on cellulose degree of polymerization. Bioresource Technol 101:7410-7415.

https://doi.org/10.1016/j.biortech.2010.04.029

19. Ilgin P, Ozay H, Ozay O (2020) Synthesis and characterization of $\mathrm{pH}$ responsive alginate basedhydrogels as oral drug delivery carrier. J Polym Res 27:251. https://doi.org/10.1007/s10965-02002231-0

20. Iwamoto S, Kai W, Isogai A, Iwata T (2009) Elastic Modulus of Single Cellulose Microfibrils from Tunicate Measured by Atomic Force Microscopy. Biomacromolecules 10:2571-2576. https://doi.org/10.1021/bm900520n

21. Ji Z, Bao X, Liu H, Wang Y, Chen L, Yu L (2017) Engineering starch-based capsules for controlled delivery. J Control Release 259:e88. https://doi.org/10.1016/j.jconrel.2017.03.191

22. Jimenez A, Fabra MJ, Talens $P$, Chiralt A (2010) Effect of lipid self-association on the microstructure and physical properties of hydroxypropyl-methylcellulose edible films containing fatty acids. Carbohyd Polym 82:585-593. https://doi.org/10.1016/j.carbpol.2010.05.014

23. Kathpalia H, Komal, Sharma, Doshi G (2014) Recent trends in Hard Gelatin capsule delivery System. J Adv Pharm Edu \& Res 4:165-177. https://doi.org/10.13140/2.1.2731.4884 
24. Ming X, Li Q, Feng J, Jiang W (2021) Analysis and optimization of process parameters of the degradable fiber mulch paper made from pineapple leaf and rice straw by response surface method. BioResources 16:3454-3468. https://doi.org/10.15376/biores.16.2.3454-3468

25. Narita T, Hébraud P, Lequeux $F$ (2005) Effects of the rate of evaporation and film thickness on nonuniform drying of film-forming concentrated colloidal suspensions. Eur Phys J E 17:69-76. https://doi.org/10.1140/epje/i2004-10109-x

26. Peng BL, Dhar N, Liu HL, Tam KC (2011) Chemistry and applications of nanocrystalline cellulose and its derivatives: A nanotechnology perspective. Can J Chem Eng 89:1191-1206. https://doi.org/10.1002/cjce.20554

27. Pharmacopeial US (2016) The United States Pharmacopeia and National Formulary USP 40-NF 35. The United States Pharmacopeia.

28. Qian L, Zhou J, Zhang L (2010) Structure and properties of the nanocomposite films of chitosan reinforced with cellulose whiskers. J Polym Sci Pol Phys 47:1069-1077. https://doi.org/10.1002/polb.21711

29. Revin V, Pestov V, Shchankin NA, Mishkin MV, Platonov VP, Uglanov VI D, A (2019) A Study of the Physical and Mechanical Properties of Aerogels Obtained from Bacterial Cellulose. Biomacromolecules 20:1401-1411. https://doi.org/10.1021/acs.biomac.8b01816

30. Rhim JW (2011) Effect of clay contents on mechanical and water vapor barrier properties of agarbased nanocomposite films. Carbohyd Polym 86:691-699. https://doi.org/10.1016/j.carbpol.2011.05.010

31. Salas C, Nypeloe T, Rodriguez-Abreu C, Carrillo C, Rojas OJ (2014) Nanocellulose properties and applications in colloids and interfaces. Curr Opin Colloid In 19:383-396. https://doi.org/10.1016/j.cocis.2014.10.003

32. Samakradhamrongthai RS, Angeli PT, Kopermsub P, Utama-ang N (2019) Optimization of gelatin and gum arabic capsule infused with pandan flavor for multi-core flavor powder encapsulation. Carbohyd Polym 226:115262. https://doi.org/10.1016/j.carbpol.2019.115262

33. Saravanakumar K, Sathiyaseelan A, Mariadoss A, Hu X, Wang MH (2020) Physical and bioactivities of biopolymeric films incorporated with cellulose, sodium alginate and copper oxide nanoparticles for food packaging application. Int J Biol Macromo 153:207-214. https://doi.org/10.1016/j.jibiomac.2020.02.250

34. Segal L, Creely JJ, Martin A Jr, Conrad C (1959) An empirical method for estimating the degree of crystallinity of native cellulose using the X-ray diffractometer Textile research journal. 29:786-794. https://doi.org/10.1177/004051755902901003

35. Siripatrawan U, Harte BR (2010) Physical properties and antioxidant activity of an active film from chitosan incorporated with green tea extract. Food Hydrocolloid 24:770-775. https://doi.org/10.1016/j.foodhyd.2010.04.003

36. Tang Y, Yang S, Zhang N, Zhang J (2014) Preparation and characterization of nanocrystalline cellulose via low-intensity ultrasonic-assisted sulfuric acid hydrolysis. Cellulose 21:335-346. 
https://doi.org/10.1007/s10570-013-0158-2

37. Xu H, Chai Y, Zhang G (2012) Synergistic Effect of Oleic Acid and Glycerol on Zein Film Plasticization. J Agr Food Chem 60:10075-10081. https://doi.org/10.1021/jf302940j

38. Yan X, Zhang K, Yang Y, Deng D, Lyu C, Xu H, Du Y (2020) Dispersible and Dissolvable Porous Microcarrier Tablets Enable Efficient Large-Scale Human Mesenchymal Stem Cell Expansion. Tissue Eng Part C-Me 26:263-275. https://doi.org/10.1089/ten.tec.2020.0039

39. Yousefhashemi SM, Khosravani A, Yousefi H (2019) Isolation of lignocellulose nanofiber from recycled old corrugated container and its interaction with cationic starch-nanosilica combination to make paperboard. Cellulose 26:7207-7221. https://doi.org/10.1007/s10570-019-02562-2

40. Zhang $Y$, Zhao Q, Wang H, Jiang $X$, Cha R (2017) Preparation of green and gelatin-free nanocrystalline cellulose capsules. Carbohyd Polym 164:358-363. https://doi.org/10.1016/j.carbpol.2017.01.096

41. Zhao J, Li S, Zhao Y, Peng Z (2020) Effects of cellulose nanocrystal polymorphs and initial state of hydrogels on swelling and drug release behavior of alginate-based hydrogels. Polym Bull 77:44014416. https://doi.org/10.1007/s00289-019-02972-z

\section{Figures}

\section{Figure 1}

Preparation of tCNC-SA film 


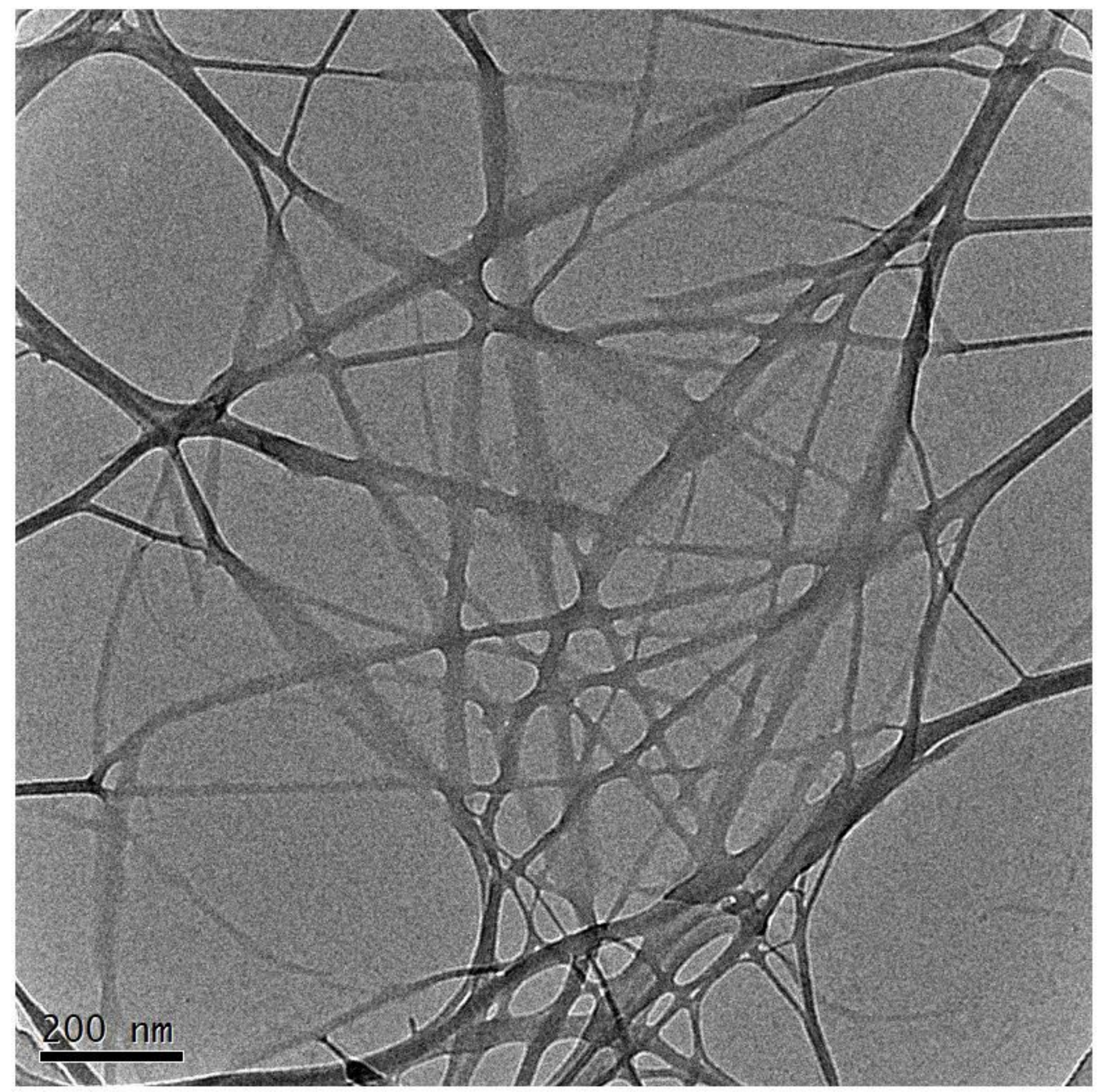

Figure 2

TEM figure of tCNC 
(a)

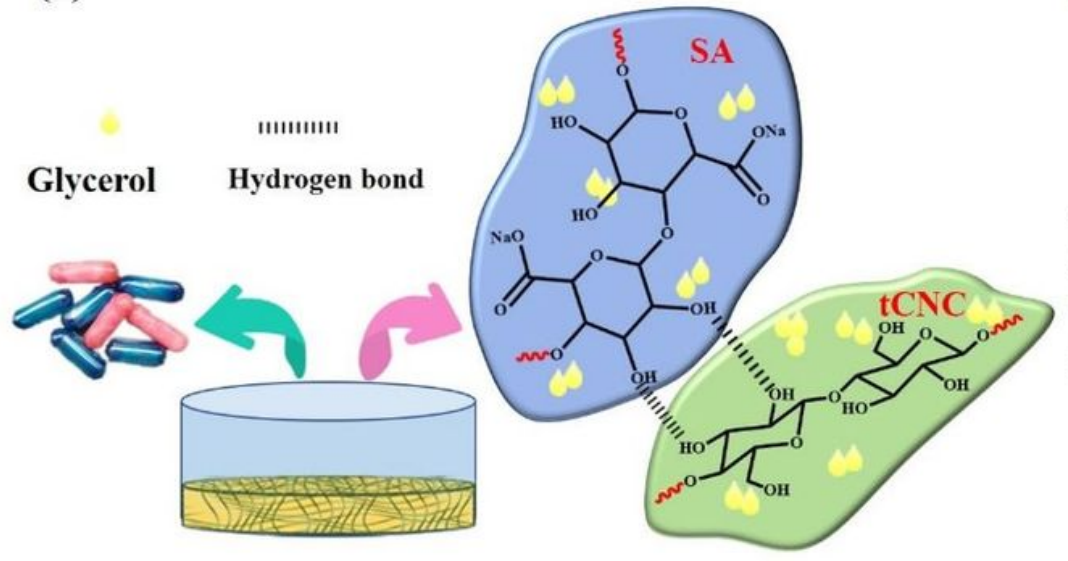

(b)

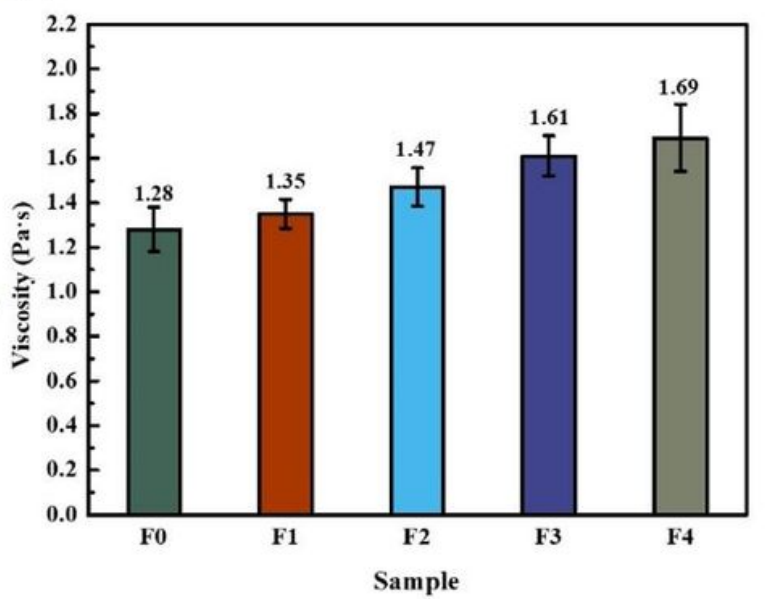

Figure 3

(a) Chemical mechanism of tCNC-SA capsule formation, (b) viscosity of different tCNC-SA gels

(a)

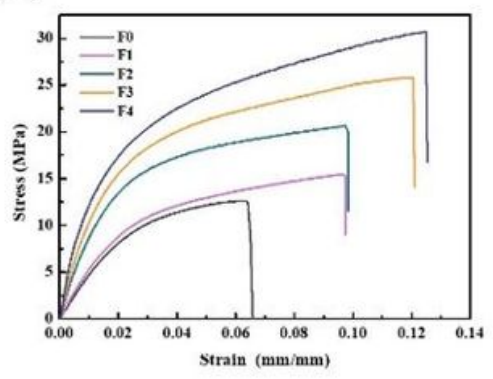

(b)

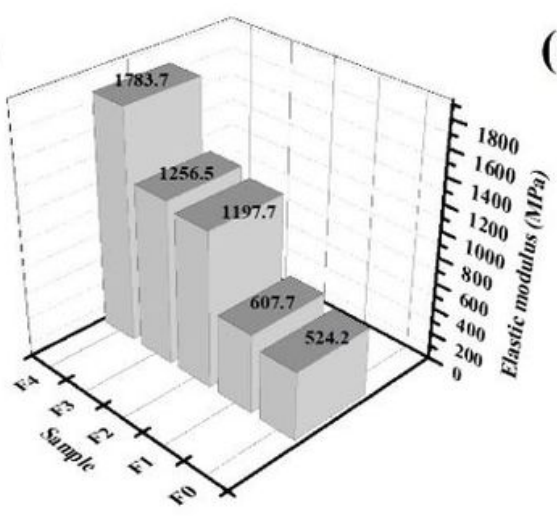

(c)

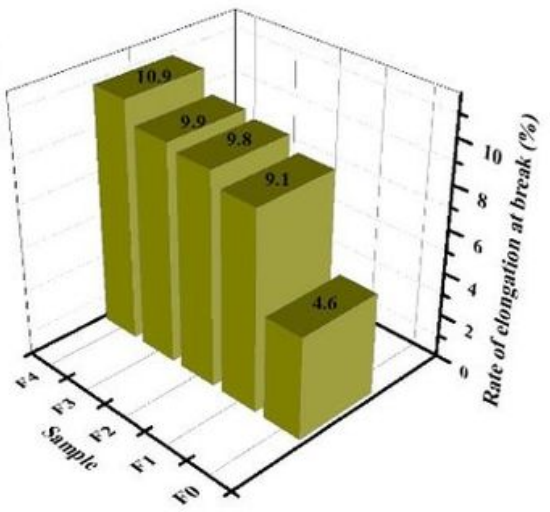

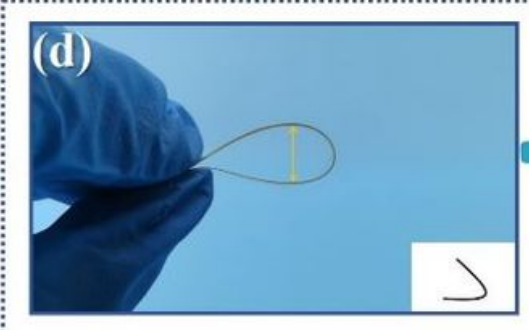
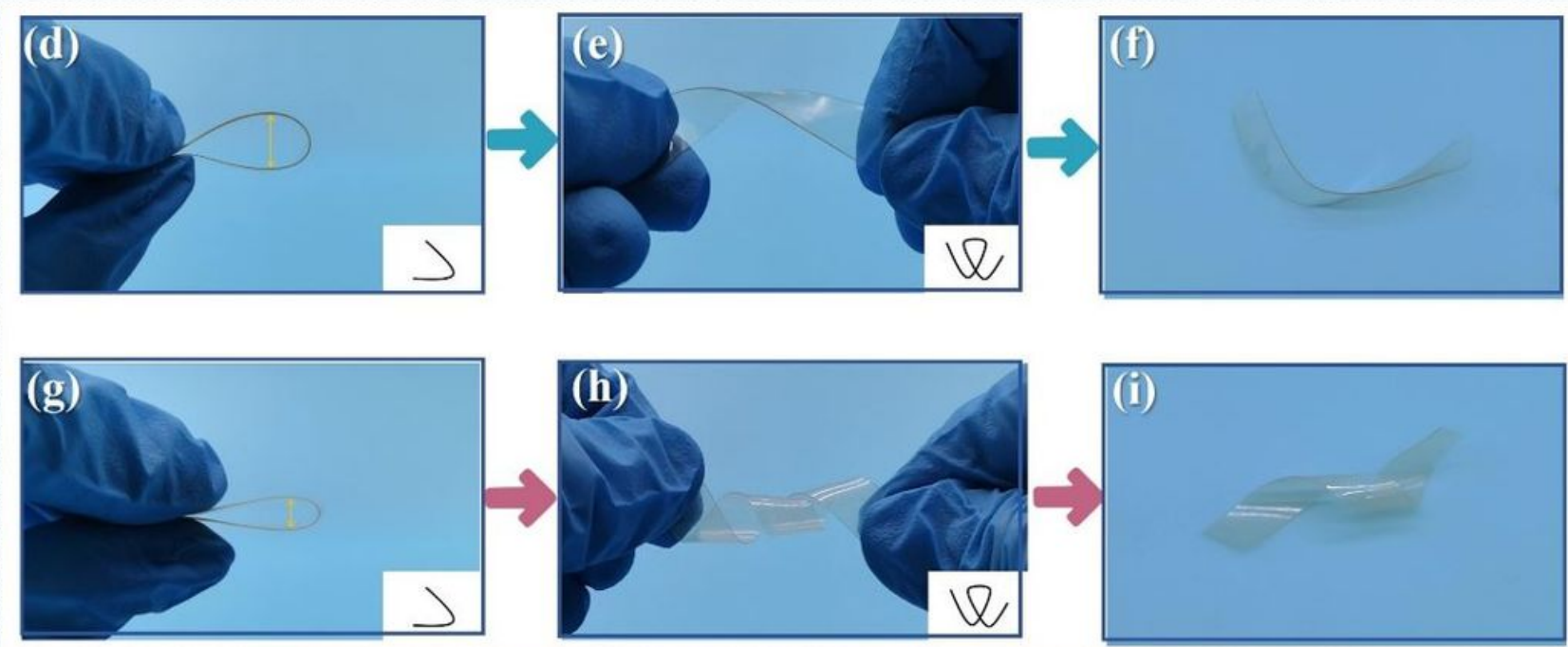

(i)

Figure 4 
(a) Stress- strain curves of tCNC-SA films, (b) Elasticity modulus of tCNC-SA films, and (c) Rate of elongation at break of tCNC-SA films, (d), (e) and (f) were flexibility of F0 film, (g) (h) and (i) were flexibility of F3 film
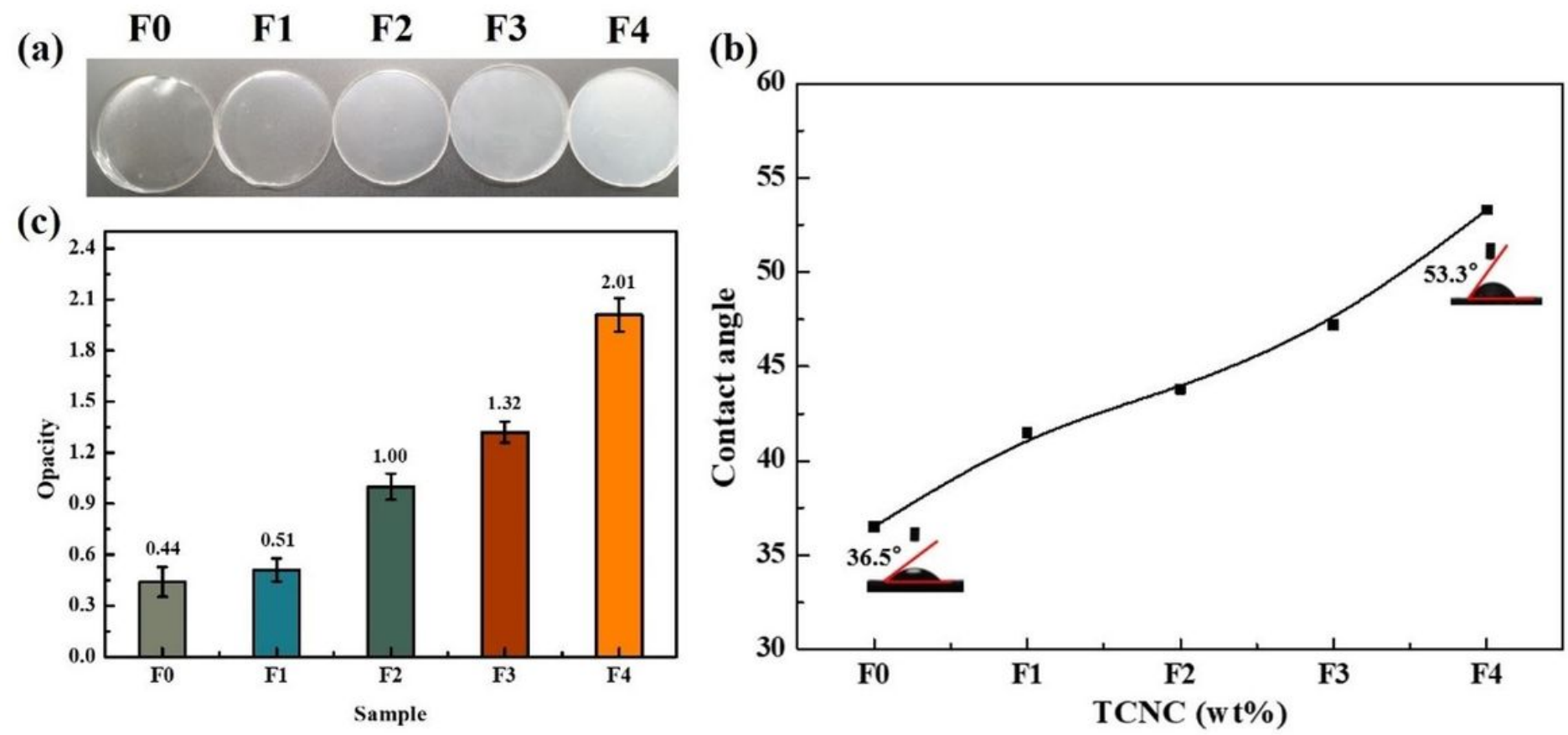

Figure 5

(a) Opacity pictures of tCNC-SA films, (b) Water contact angle of tCNC-SA films (c) Opacity data of tCNCSA films
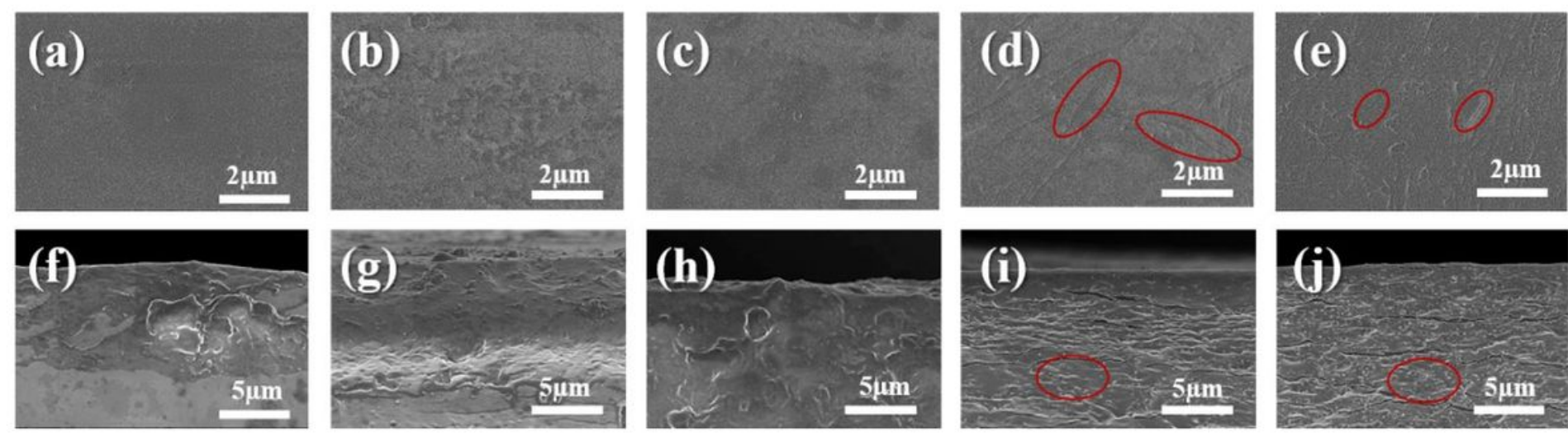

\section{Figure 6}

SEM figures of different tCNC-SA film: (a-e) surface figures of F0 - F4; (f-j) section figures of F0 - F4 

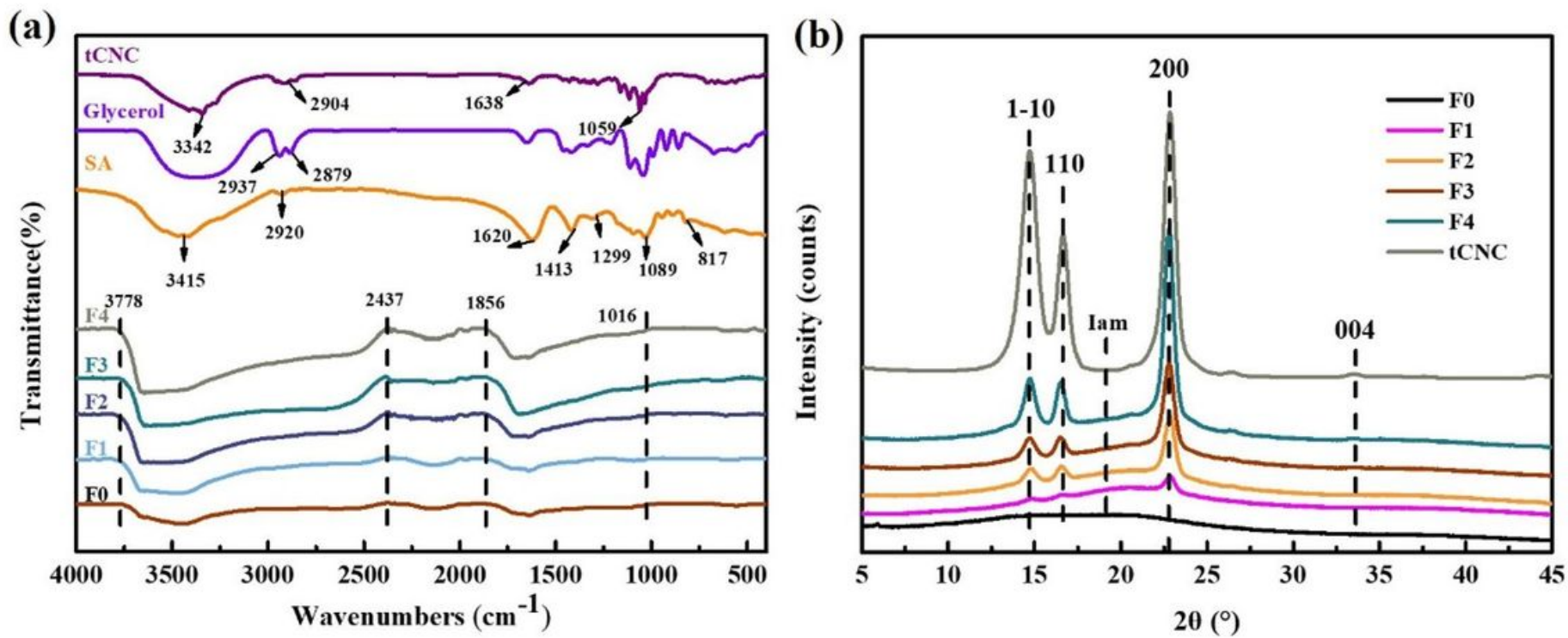

Figure 7

(a) FT-IR spectrum of different tCNC-SA film, SA and glycerol, (b) XRD graphs of different tCNC-SA films and tCNC

(a)

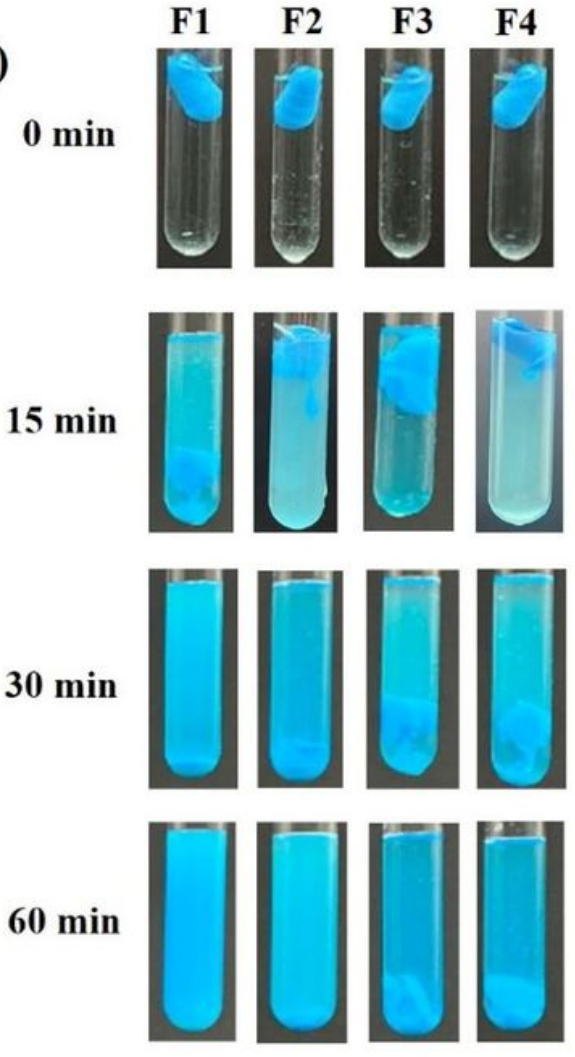

(b)

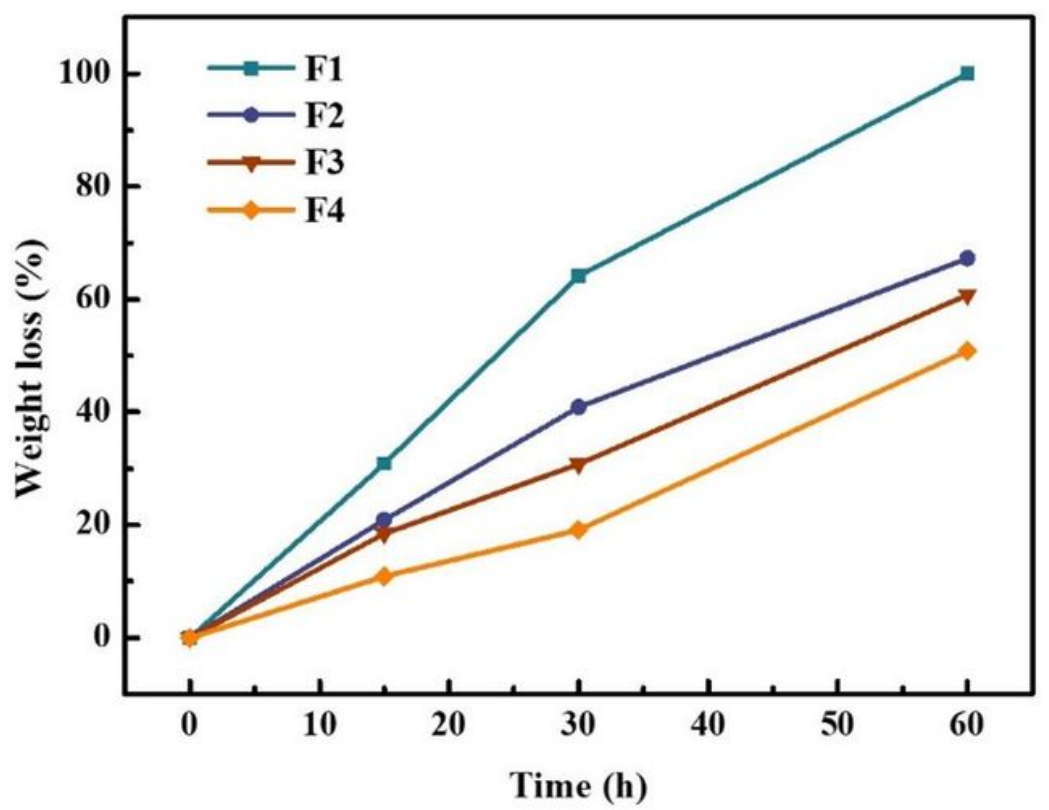

Figure 8

(a) Picture of degradation of tCNC-SA capsules (F1-F4) in 0 min, 15 min, 30 min, 60 min (b) Weight loss of tCNC-SA capsules (F1-F4) in pH 6.8 at $37^{\circ} \mathrm{C}$ 
(a)

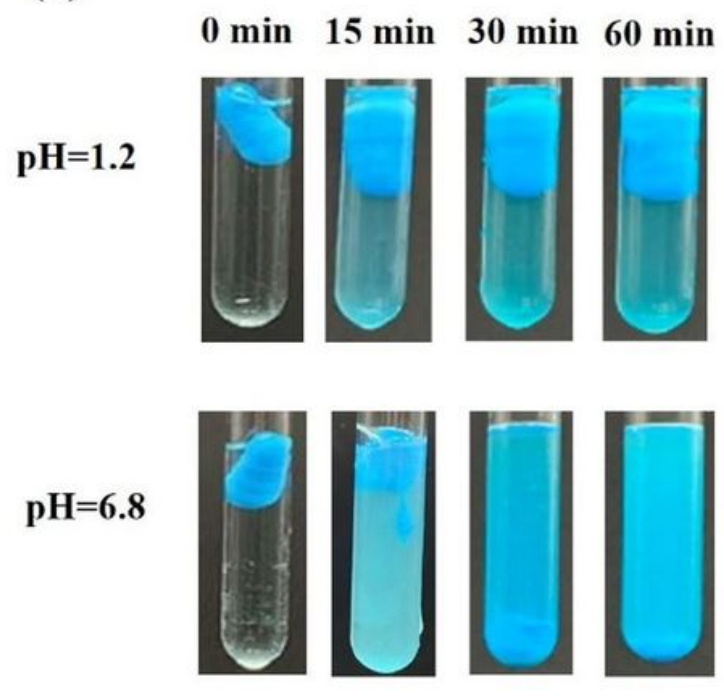

(b)

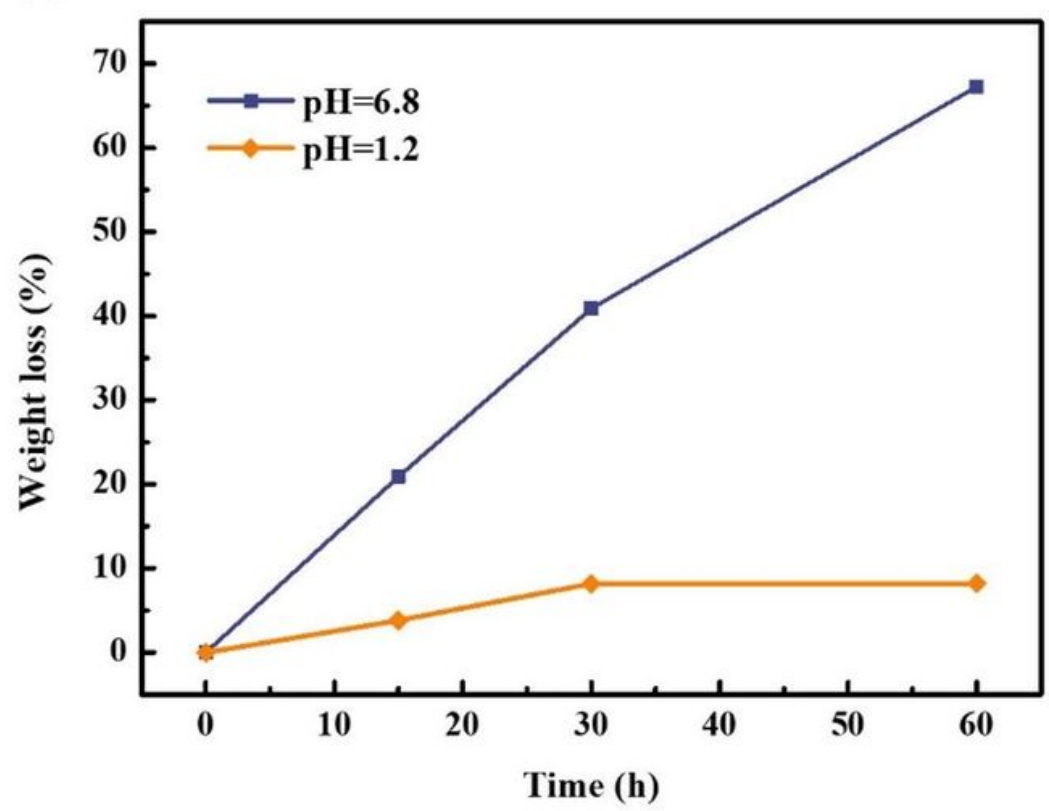

Figure 9

(a) Degradation of F2 tCNC-SA capsules at pH 1.2 and pH 6.8, (b) Weight loss of F2 tCNC-SA capsules at $\mathrm{pH} 1.2$ and $\mathrm{pH} 6.8$

\section{Supplementary Files}

This is a list of supplementary files associated with this preprint. Click to download.

- Graphicalabstract.jpg 